- Prof. Dr. Ute Sacksofsky, Universität Frankfurt, 2002: Steuerung der Familie durch Steuern. http://www.fernuni-hagen.de/videostreaming/zmi/video/2002/02-15_00000/

- Prof. Dr. Monika Frommel, Universität Kiel, und Prof. Dr. Jan Beckmann, FernUniversität in Hagen, 2012: Rechtliche und ethische Fragen der Reproduktionsmedizin. http://www. fernuni-hagen.de/videostreaming/gleichstellung/201205/

Geplant u.a.

- Ulrike Spangenberg, Freie Universität Berlin: Geschlechtergerechtigkeit im Steuerrecht?

Die Interviews im Portal „Recht und Gender“ sind frei zugänglich. Damit soll für das Gendermodul geworben und Studierenden der anderen Fakultäten und Studiengänge sowie externen Interessierten die Möglichkeit zur Bearbeitung der Inhalte und damit Stärkung ihrer Genderkompetenz im Recht geboten werden.
Über das Internetportal „Recht und Gender“ werden auch Video-Interviews mit Portraits herausragender Rechtswissenschaftlerinnen zugänglich gemacht. Das erste war mit Richterin am Bundesverfassungsgericht Prof. Dr. Susanne Baer. Die Porträts werden im Kontext des Projekts JurPro aufgezeichnet. JurPro - De jure und de facto: Professorinnen in der Rechtswissenschaft - ist ein vom BMBF über die Förderlinie Frauen an die Spitze gefördertes Drittelmittelprojekt. Es sollen die Bedingungen von Professorinnenkarrieren untersucht und Vorschläge zur Verbesserung der Organisationsstruktur und -kultur in den Rechtswissenschaften erarbeitet werden. vgl. www.fernuni-hagen.de/jurpro

\section{Ansprechpartnerin}

AOR'Ulrike Schultz

Tel.: 02331-870811

Ulrike.Schultz@FernUni-Hagen.de

\title{
Rezension: Festschrift 150 Jahre djt
}

\author{
150 Jahre Deutscher Juristentag \\ Festschrift Deutscher Juristentag 1860-2010 \\ Festschriften \\ 2010. Buch. XVIII, 763 S. In Leinen \\ C.H.BECK ISBN 978-3-406-59824-1 \\ München 2010 \\ 168,oo Euro inkl. MwSt.
}

Die Festschrift zum 150-jährigen Jubiläum des Deutschen Juristentages (djt) zeichnet ein sowohl sehr umfassendes Bild der inhaltlichen Arbeit und des Einflusses des djt als auch der Vereinsgeschichte und des Vereinslebens. Das umfangreiche Werk beginnt mit einer selbstkritischen Auseinandersetzung mit der Geschichte des Verbandes. Insbesondere wird die stillschweigende Akzeptanz nach 1945 von Juristen in den eigenen Reihen, denen eine nationalsozialistische Gesinnung nachgewiesen werden konnte, beleuchtet. Im Anschluss wird auf über 400 Seiten zum Teil sehr ausführlich für jedes Rechtsgebiet die inhaltliche Arbeit des Juristentages dargestellt. Der dritte Teil der Festschrift befasst sich mit der Außensicht, während sich der vierte und letzte Teil mit der Binnensicht auf den djt beschäftigt.

Für aktive Vereinsmitglieder ist diese Festschrift sicherlich empfehlenswert, für alle anderen ist sie außerordentlich detailliert. Aus Genderperspektive ist zu bedauern, dass trotz zahlreicher ak- tiver Juristinnen im djt nur zwei der 30 Autor/inn/en weiblich sind. Gleichberechtigung und Gleichstellung kommen als Themen fast nur punktuell im Zusammenhang mit Beschlüssen des djt vor. Die frauen- bzw. geschlechterrelevanten Beschlüsse des djt sind jedoch aus frauenrechtspolitischer Sicht nicht immer begrüßenswert. Um nur zwei Beispiele zu nennen: Der djt sprach sich im Jahr 1974 gegen ein Verbot der Frage nach einer Schwangerschaft im Vorstellungsgespräch aus. 30 Jahre später plädierte der djt für eine Abschaffung des Anspruchs auf Teilzeit im Teilzeit- und Befristungsgesetz.

Ein frauenrelevantes Thema, das an verschiedenen Stellen des Buches diskutiert wird, sind die Diskussionen 2000 und 2002 zur Fassung der Satzung in geschlechtergerechter Sprache. Die geschlechtsneutrale Satzung wurde maßgeblich von der damaligen 1 . Vorsitzenden des djb, Ursula Nelles, und weiteren djb-Kolleginnen gefordert. Reinhard Böttcher, Präsident des djt 2000 und 2002, erwähnt in seinem Beitrag, was für eine peinliche Berichterstattung er gefürchtet hatte, wenn die Abstimmung über die neue Fassung der Satzung in geschlechtergerechter Sprache 2002 gescheitert wäre.

Es bleibt für die Zukunft zu hoffen, dass der djt es bei zukünftigen Veröffentlichungen auch als Blamage ansehen wird, für ein solches Werk weniger als zehn Prozent Autorinnen vorweisen zu können. (Elif Beden) 